\title{
Absorption of Inhaled Antigen into the Circulation of Isolated Lungs from Normal and Immunized Rabbits
}

\author{
Janet F. Braley, Christopher A. Dawson, Vernon L. Moore, and Bruce O. \\ CozzInI, Research Service, Wood Veterans Administration Center, and the \\ Departments of Medicine, Physiology, and Pathology, The Medical College \\ of Wisconsin, Milwaukee, Wisconsin 53226
}

A B S T R A C T The purpose of this study was to determine whether the absorption of inhaled antigen $(\mathrm{Ag})$ across the pulmonary air-blood barrier of the isolated perfused lung can be modulated by immunologic mechanisms. Lungs from immunized or nonimmunized rabbits were removed, ventilated, and perfused with autochthonous blood. Radioiodinated Ag (human serum albumin or ovalbumin) was introduced as an aerosol into the isolated lung for $15 \mathrm{~min}$ and blood samples were taken over a 4 -h period. The results showed that radioactivity from inhaled $\mathrm{Ag}$ entered the perfusing blood as two fractions. One fraction was precipitable by $5 \%$ trichloroacetic acid or antiserum. The TCA-soluble fraction chromatographed differently from iodide and may have represented metabolites of the Ag. Immunization specifically reduced the amount of antigenically intact protein entering the blood. On the other hand, the metabolite reached higher concentrations in the blood of immunized lungs. We conclude that the alveolar capillary barrier of the normal rabbit lung could provide a significant route of entry for inhaled antigen into the systemic circulation and that immunization reduces absorption via this route and enhances pulmonary metabolism of the Ag.

\section{INTRODUCTION}

Little is known about the mechanisms by which inhaled soluble antigens enter the circulation. Since the availability of isotopic techniques, it is generally agreed that proteins can cross the alveolar-capillary barrier of the lung antigenically intact $(1,2)$. However, the quantitative importance and mechanisms of uptake via this route are not clear (3).

Dr. Braley is the recipient of a National Research Service Institutional Award, AL 7006.

Received for publication 15 April 1977 and in revised form 27 December 1977.
There is some evidence indicating that immunization may alter the absorption of antigen from the lung (4). In addition, studies from our laboratory by Thrall et al. (5) have shown that after intratracheal administration of ovalbumin (OA), ${ }^{1}$ lower levels of the antigen were found in the blood of OA-immunized rabbits than in nonimmunized animals. The phenomenon was immunospecific and was transferable to normal animals with immune serum. The results also suggested that at least part of this difference was due to decreased antigen absorption from the lung.

In the present study, we have used an isolated perfused rabbit lung preparation to investigate the absorption of protein across the air-blood barrier and to determine whether the uptake of antigen by this route can be modulated by an immunologic mechanism.

\section{METHODS}

Animals and immunization. New Zealand white rabbits of either sex, weighing $2-4 \mathrm{~kg}$, were immunized subcutaneously in two inguinal sites with $2 \mathrm{mg}$ of human serum albumin (HSA), bovine gamma globulin (BGG), or OA in complete Freund's adjuvant, and were used 25-33 days after immunization.

Serum antibody titers were determined by passive hemagglutination (6). The solution of BGG had some sheep erythrocyte-agglutinating activity, therefore, it was absorbed with sheep erythrocytes before coating the tanned cells.

Antigens. HSA and OA were obtained from Miles Laboratories (Kankakee, Ill.), rabbit serum albumin from N. L. Cappel Laboratories Inc. (Cochranville, Pa.), complete Freund's adjuvant from Difco Laboratories (Detroit, Mich.), and HSA labeled with ${ }^{131}$ I (RISA) from Mallinckrodt Inc. (St. Louis, Mo.). ${ }^{125}$ I-Sodium was obtained from Amersham Corp. (Arlington Heights, Ill.), and used to label OA and rabbit serum albumin by the potassium monochloride method of Bale et al. (7). The free ${ }^{125} \mathrm{I}$ was removed by extensive dialysis against physiological saline.

Isolated perfused lung preparations. The lung prepara-

${ }^{1}$ Abbreviations used in this paper: BGG, bovine gamma globulin; HSA, human serum albumin; OA, ovalbumin; RISA, HSA labeled with ${ }^{131} \mathrm{I}$. 
tion has been described by Hakim et al. (8). The anesthetized animal was exsanguinated through a carotid arterial catheter after the administration of heparin (2,000 U). $20 \mathrm{ml}$ of dextran 40 (Rheomacrodex, Pharmacia Inc., Piscataway, N. J.) was administered during the bleeding process. Autochthonous blood $(120 \mathrm{ml})$ was used to prime the perfusion system. The trachea was cut $3 \mathrm{~cm}$ above the bronchial bifurcation. The pulmonary artery and left atrium were cannulated, the heart ligated, and the lung placed in a negative pressure ventilation chamber supported by a tracheal cannula.

The lung was ventilated at a frequency of 10 per minute with a minimum inspiratory pressure of $-10 \mathrm{~cm}_{2} \mathrm{O}$, and an end-expiratory pressure of $-2 \mathrm{~cm} \mathrm{H}_{2} \mathrm{O}$. At 15-min intervals, a deep inspiration was produced by decreasing the inspiratory pressure to $-22 \mathrm{~cm} \mathrm{H}_{2} \mathrm{O}$ for one breath. The tracheal cannula was connected to a meteorological balloon containing a gas mixture of $\cong 16 \% \mathrm{O}_{2}, 6 \% \mathrm{CO}_{2}$, and $78 \% \mathrm{~N}_{2}$. This maintained the blood $\mathrm{PO}_{2}, \mathrm{PCO}_{2}$ at $\cong 100$ and $40 \mathrm{~mm} \mathrm{Hg}$, respectively, and the $\mathrm{pH}$ at 7.4. The blood, warmed to $37^{\circ} \mathrm{C}$, was pumped from a reservoir into the pulmonary artery by a roller pump at a rate of $160 \mathrm{ml} / \mathrm{min}$. The blood then drained from the left atrium back into the reservoir. Arterial and venous pressures (relative to the height of the left atrium) were measured throughout each experiment from pressure taps in the perfusion cannulas. Pulmonary venous pressure was set at $-4 \mathrm{~mm} \mathrm{Hg}$ and held constant. The volume of blood in the perfusion circuit was monitored throughout each experiment by measuring the height of blood in the reservoir.

Insufflation of antigen. Radioiodinated antigens were dialyzed overnight at $4^{\circ} \mathrm{C}$ against physiological saline just before use. Approximately $99 \%$ of ${ }^{131}$ I from RISA, ${ }^{125}$ I from rabbit serum albumin, or $95 \%$ of ${ }^{125} \mathrm{I}$ from $\mathrm{OA}$ was protein bound, as determined by precipitation with trichloroacetic acid (TCA). After the perfusion system and reservoir volumes had stabilized $(\sim 15 \mathrm{~min}$ after beginning the isolated perfusion), antigen was introduced into the lung as an aerosol. This was accomplished by placing $\sim 100 \mu \mathrm{Ci}$ of RISA or ${ }^{125}$ I-rabbit serum albumin, or $25 \mu \mathrm{Ci}$ of ${ }^{125} \mathrm{I}$-OA diluted in $5 \mathrm{ml}$ of physiological saline into an ultrasonic nebulizer (DeVilbiss 65, Somerset, Pa.). The nebulizer was operated at the maximum rate (vapor particles from 2 to $10 \mu \mathrm{m}$ in diameter [9]), while the aerosol and respiratory gases were rebreathed for $15 \mathrm{~min}$.

Collection of samples and calculations. 1-ml blood samples were taken from the reservoir at 30 -min intervals for $4 \mathrm{~h}$ after the beginning of antigen inhalation. Total counts per minute (cpm) which had entered the blood by the end of each time interval were calculated from the activity of the sample and the circulating blood volume corrected for volume lost in sampling. At the end of the experiment, the trachea and extraparenchymal bronchi were trimmed from the lung. The lungs were weighed and cut into slices ( $\cong 1 \mathrm{~g}$ ) for measurement of radioactivity. Total activity which had reached the parenchyma, including smaller airways, was determined by adding the counts per minute found in the blood and in the lung at the end of the experiment. After weighing, two normal and two HSA-immunized lungs were lavaged by injecting $20 \mathrm{ml}$ of physiological saline, intratracheally.

The blood samples were centrifuged at 2,000 $\mathrm{g}$ for $15 \mathrm{~min}$ and the plasma removed. Cells were routinely washed three times with five volumes of saline; $<1 \%$ of the counts per minute remained with the cells.

TCA precipitation was carried out on plasma from each blood sample, on samples of the inhaled antigen before and after nebulization, and on lavage fluids. Radioactivity of all samples was measured in a Beckman model gamma 300 radiation counter (Beckman Instruments, Inc., Fullerton, Calif.). The data are reported as percent of inhaled counts per minute entering the blood in the TCA-precipitable and TCA-soluble fractions as a function of time, and the mean values of experimental groups compared by Student's unpaired $t$ test.

Studies on TCA fractions. Precipitation by TCA was carried out at a final concentration of $12 \%$ plasma and $5 \%$ TCA. Samples were centrifuged at $2,000 \mathrm{~g}$ for $30 \mathrm{~min}$ at $4^{\circ} \mathrm{C}$, and the activity in the supernatant fluid was compared to total activity.

Antigen-associated radioactivity in the plasma was assessed by coprecipitation with pooled rabbit antiserum and antigen at equivalence. $0.5 \mathrm{ml}$ of rabbit anti-HSA or anti-OA serum was added to an equal volume of postperfusion plasma. Antigen at equivalence was added in a volume of $0.1 \mathrm{ml}$ saline and complexes were allowed to form at $37^{\circ} \mathrm{C}$ for $1 \mathrm{~h}$ and $4^{\circ} \mathrm{C}$ for $24 \mathrm{~h}$. After centrifugation at $2,000 \mathrm{~g}$ for $30 \mathrm{~min}$, the radioactivity in the supernate was compared to total activity.

Column chromatography was performed by loading $2 \mathrm{ml}$ of post-perfusion plasma from lungs which had been insufflated with RISA onto a $0.7 \times 10.8 \mathrm{~cm}$ Sephadex G-10 column (Pharmacia Inc.) equilibrated with $0.01 \mathrm{M}$ phosphate-buffered saline, $\mathrm{pH}$ 7.4. The column was eluted at a flow rate of $0.2 \mathrm{ml} / \mathrm{min}$ at room temperature. Blue dextran and ${ }^{125} \mathrm{I}-\mathrm{Na}$ were included as standards. The high molecular weight fraction of ${ }^{131} \mathrm{I}$ was eluted with blue dextran (void volume) in phosphate-buffered saline, followed by a distilled water wash which eluted a single peak of radioactivity containing both ${ }^{131} \mathrm{I}$ and ${ }^{125} \mathrm{I}$.

The low molecular weight fraction was further chromatographed by streaking $0.2 \mathrm{ml}$ onto Gelman ITLC type SG paper (Gelman Instrument Co., Ann Arbor, Mich.). $5 \mu \mathrm{l}$ of 3-iodotyrosine (Sigma Chemical Co.) in ethanol was spotted at the origin as a standard and the chromatogram developed in ethyl acetate, methanol, and ammonia (50:25:0.5, vol:vol) to a height of $\cong 16 \mathrm{~cm}$. The dried chromatogram was exposed to ammonia vapor for $1 \mathrm{~min}$ and then sprayed with fluorescamine (Fluram, Roche Diagnostics Div., Hoffman-La Roche Inc., Nutley, N. J.) in acetone $(0.1 \mathrm{~g} / 100 \mathrm{ml})$. Iodotyrosine appeared as a yellow spot under long-wave ultraviolet illumination. Strips ( $1 \mathrm{~cm}$ wide) were cut along the width of the chromatogram and the radioactivity of both isotopes was estimated in the gamma counter.

\section{RESULTS}

Isolated lungs insufflated with RISA. Isolated perfused lungs from 7 normal, 11 HSA-immunized and 5 BGG-immunized rabbits were insufflated with RISA, as described in Methods. The lungs were judged as being in good condition throughout the 4 -h experimental period because there was little loss of blood from the reservoir to the lungs, no gross pulmonary edema was observed, and the lungs appeared to collapse completely at the end of each experiment. The mean lung weight to body weight ratio at the end of the perfusion period was $3.4 \mathrm{~g} / \mathrm{K} \pm 0.1 \mathrm{SE}$, which compares to a ratio of $3.6 \mathrm{~g} / \mathrm{K}$ for freshly killed rabbits in our laboratory, a further indication that edema was minimal. The mean pulmonary arterial pressure was $7.0 \mathrm{~mm} \mathrm{Hg} \pm 0.4 \mathrm{SE}$ before antigen insufflation and rose to $11.4 \mathrm{~mm} \mathrm{Hg} \pm 0.7 \mathrm{SE}$ at the end of $3.5 \mathrm{~h}$. In four lungs (one normal and three HSA-immunized), 
the pressures began to rise precipitously between 3.5 and $4 \mathrm{~h}$, at which time the experiments were terminated. There was no statistically significant difference in mean pulmonary arterial pressure or lung weight to body weight ratio among the experimental groups.

The amount of vapor inspired during the period of antigen insufflation was $0.44 \pm 0.03 \mathrm{ml} \mathrm{SE}$, containing a mean of $955 \mathrm{~g} \pm 112 \mu \mathrm{g}$ SE protein. In one normal experiment, a higher concentration of antigen was used so that $3.9 \mathrm{mg}$ HSA in $0.34 \mathrm{ml}$ vapor was inhaled. Antigen absorption from this lung, normalized to dose, was no less than in other normal lungs, indicating that the absorption process was not saturated over the dose range studied.

The geometric mean serum antibody titer (as determined by passive hemagglutination) for the HSAimmunized group was $1: 3,200$, and for the BGGimmunized group was $1: 1,690$. There was no crosshemagglutination between anti-HSA and anti-BGG antisera at a dilution of 1:10 when erythrocytes were coated with the heterologous antigen.

Appearance of TCA-precipitable and TCA-soluble ${ }^{131}$ I in the circulation of normal, HSA-immunized and BGG-immunized lung preparations. Fig. 1 shows the appearance of TCA-precipitable and nonprecipitable fractions of ${ }^{131} \mathrm{I}$ in the blood of 7 normal, 11 HSAimmunized and 5 BGG-immunized lung preparations over a 4 -h period after insufflation with RISA.

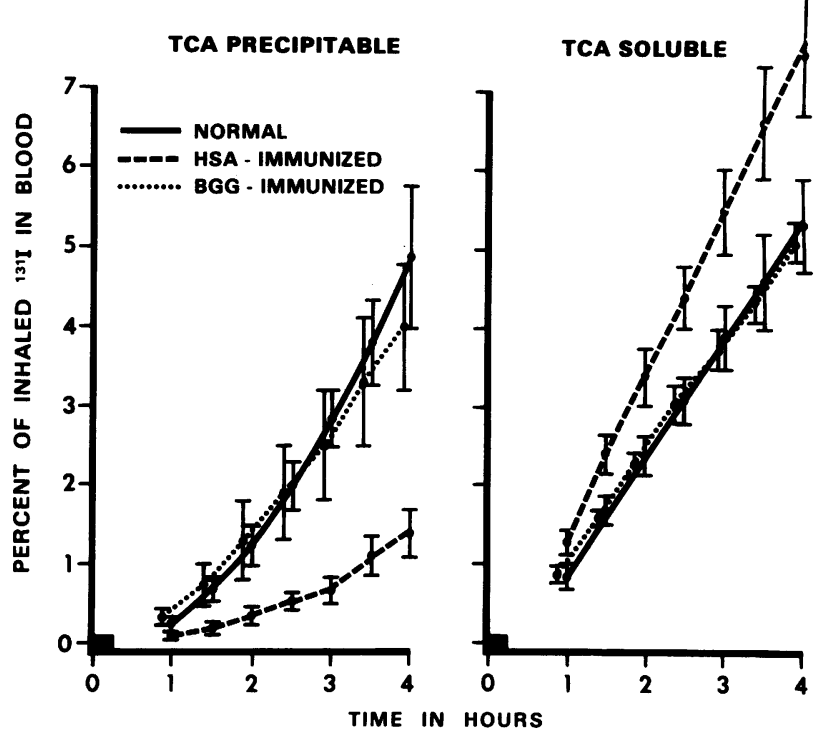

FIGURE 1 Time-course of the appearance of TCA-precipitable and TCA-soluble ${ }^{131} \mathrm{I}$ in blood of isolated perfused lung preparations. The dark bar represents 15 min of insufflation with RISA. The ordinate is percent of inhaled dose of ${ }^{131} \mathrm{I}$ which was present in the blood in each TCA fraction. Each point represents the mean $\pm S E$ for preparations from 7 normal, 11 HSA-immunized, and 5 BGG-immunized animals (immunospecificity control).
The TCA-precipitable (protein associated) ${ }^{131} \mathrm{I}$ entered the blood slowly for the 1st h, then appeared more rapidly in both normal and HSA-immunized preparations. The mean blood levels of the TCAprecipitable fraction were significantly different $(P$ $<0.005)$ between these two groups from $1.5 \mathrm{~h}$ and beyond. At $4 \mathrm{~h}$, the blood of the normal control contained $4.9 \pm 0.9 \% \mathrm{SE}$ of inhaled ${ }^{131}$ I bound to protein, whereas, in the HSA-sensitized group only $1.4 \pm 0.3 \%$ $\mathrm{SE}$ of the inhaled dose was found in the protein bound fraction $(P<0.001)$.

The TCA-soluble (nonprotein associated) fraction of ${ }^{131}$ I entered the blood at a constant rate. At the end of $4 \mathrm{~h}$, this fraction accounted for $5.3 \pm 0.6 \% \mathrm{SE}$ of inhaled ${ }^{131} \mathrm{I}$ in normal, and $7.5 \pm 0.7 \% \mathrm{SE}$ in HSAimmunized preparations. The difference in the amount of soluble ${ }^{131}$ I in the blood of normal and HSA-immunized preparations was statistically significant $(P$ $<0.05$ ) from $2 \mathrm{~h}$ and beyond.

As a specificity control, five rabbits were immunized with a different antigen, BGG, and challenged with HSA. TCA-precipitable and TCA-soluble ${ }^{131}$ I appeared in the blood of these controls at rates similar to those seen in the normal, nonimmunized controls. At $4 \mathrm{~h}$, the blood of BGG-immunized preparations contained $4.0 \pm 0.8 \% \mathrm{SE}$ of inhaled ${ }^{131} \mathrm{I}$ in the TCA-precipitable fraction and $5.1 \pm 0.3 \% \mathrm{SE}$ in the TCA-soluble fraction.

The results suggest that immunization with HSA caused an appreciable reduction in the movement of the TCA-precipitable fraction of inhaled RISA into the blood of the isolated lung preparation. They also suggest that a larger quantity of TCA-soluble ${ }^{131} \mathrm{I}$ entered the blood of the immune preparation.

Characterization of TCA-fractions of plasma ${ }^{131} I$. The same fraction of plasma ${ }^{131} \mathrm{I}$ precipitated by TCA was precipitated by anti-HSA and was also nondialyzable (Table I). Therefore, this fraction was antigenically intact and probably did not contain a significant amount of ${ }^{131} \mathrm{I}$ associated with other serum proteins.

The TCA-soluble fraction of ${ }^{131}$ I was eluted from a Sephadex G-10 column in a single peak which also

TABLE I

Comparison of Dialyzable, TCA-Precipitable, and Antiserum-Precipitable Fractions of Radioactive Iodine in Blood of Nonimmune Lungs $4 h$ after Insufflation with Antigen

\begin{tabular}{lcc}
\hline \multicolumn{1}{c}{ Antigen inhaled } & HSA & OA \\
\hline & $\%$ & $\%$ \\
Total cpm in l-ml plasma & $2,806(100)$ & $1,726(100)$ \\
Nondialyzable cpm & $1,468(52)$ & $317(18)$ \\
TCA-precipitable cpm & $1,454(52)$ & $311(18)$ \\
Anti-HSA-precipitable cpm & $1,394(50)$ & $0(0)$ \\
Anti-OA-precipitable cpm & $8(0.3)$ & $278(16)$ \\
\hline
\end{tabular}




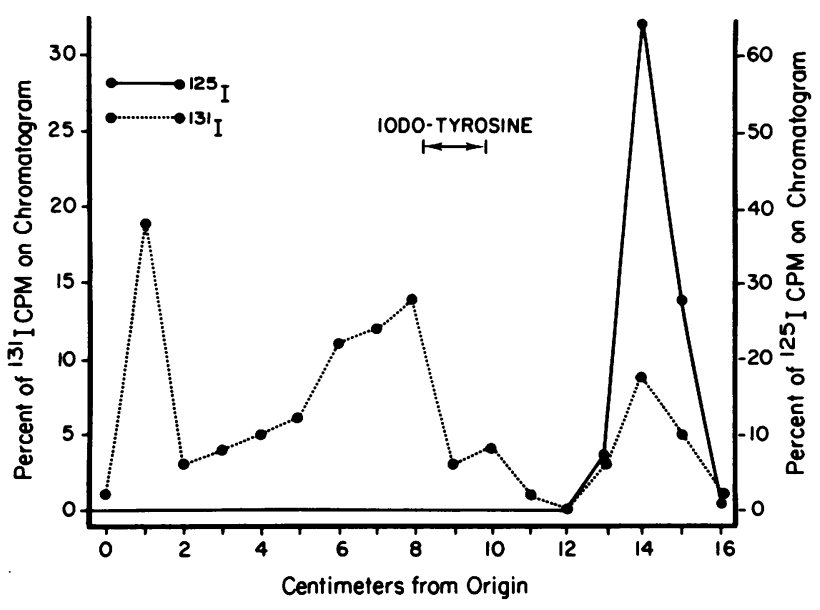

FIGURE 2 Results of thin-layer chromatography of the TCAsoluble fraction of blood ${ }^{131}$ I $4 \mathrm{~h}$ after insufflation with RISA. ${ }^{125} \mathrm{I}-\mathrm{Na}$ and 3-iodotyrosine were added as markers. Each point represents the percent of total ${ }^{131} I$ or ${ }^{125} I$ in a $1-\mathrm{cm}$ strip, and the arrow represents the zone of migration of iodotyrosine.

included a ${ }^{125}$ I standard. Fig. 2 shows the results of thinlayer chromatography of a sample from this peak. Approximately $80 \%$ of the TCA-soluble ${ }^{131}$ I of plasma had mobilities different from the 125-labeled sodium iodide standard. Similar results were obtained with plasma from normal or immunized animals, therefore most of the TCA-soluble fraction of ${ }^{131} \mathrm{I}$ present in the blood after $4 \mathrm{~h}$ of perfusion was associated with small molecules which did not cochromatograph with free iodide. Thinlayer chromatography of RISA before dialysis showed that all of the TCA-soluble ${ }^{131}$ I was iodide. The quantity of radioactivity associated with free iodide in the blood was no more than could be accounted for on the basis of rapid equilibration of that present in the inhaled RISA.

Site of dissociation of TCA-soluble ${ }^{131}$ I from RISA. Table II shows mean TCA-precipitable fractions from several sites in the experimental preparation. To assure that dissociation of ${ }^{131}$ I from RISA was not caused by the ultrasonic nebulizer, TCA-precipitation was routinely done on pre- and postnebulized RISA. In addition, four lungs were lavaged to determine whether TCA-soluble ${ }^{131}$ I could be washed from the air side of the lung; <2\% TCA-soluble ${ }^{131}$ I was found in these sites. To determine whether the dissociation could have occurred after the antigen entered the blood, experiments were performed in which RISA was added to the circulating blood of normal lungs and allowed to perfuse the lung for $4 \mathrm{~h}$. Again, no increase in the TCA-soluble ${ }^{131}$ I fraction could be detected.

Isolated lungs insufflated with OA. To determine whether the immunologic modification of antigen absorption extended to another antigen, ${ }^{125} \mathrm{I}-\mathrm{OA}$ was insufflated into isolated lungs from nine rabbits im-
TABLE II

TCA-Precipitable Fraction of ${ }^{131} \mathrm{I}$ in Aerosol, Lung Lavage, and Blood $4 h$ after Insufflation with RISA $( \pm S E)$

\begin{tabular}{lrc}
\hline \multicolumn{1}{c}{ Site of sampling } & $\begin{array}{c}\text { Number of } \\
\text { experiments }\end{array}$ & $\begin{array}{c}\text { TCA- } \\
\text { precipitable } \\
{ }^{131}\end{array}$ \\
\hline & & $\% \pm S E$ \\
Prenebulized RISA & 22 & $99.2 \pm 0.1$ \\
Postnebulized RISA & 22 & $99.1 \pm 0.1$ \\
Lung lavage fluid* & 4 & $98.5 \pm 0.6$ \\
Blood after antigen inhalation & & \\
$\quad \begin{array}{l}\text { Normal } \\
\quad \text { HSA-immunized }\end{array}$ & 7 & $48.0 \pm 3.0$ \\
$\quad$ BGG-immunized & 10 & $17.0 \pm 3.9$ \\
Blood after antigen was introduced & 5 & $43.0 \pm 4.5$ \\
$\quad$ into circulation of normal lungs & 2 & 98.9 \\
\hline
\end{tabular}

* Two normal, two HSA-immunized.

munized with $\mathrm{OA}$ and nine controls immunized with HSA. The amount of vapor inspired was $0.34 \pm 0.15 \mathrm{ml}$. Due to the higher specific activity of ${ }^{125} \mathrm{I}$ on OA, the mean amount of inspired protein was $5.7 \mu \mathrm{g}$ (as compared to $955 \mu \mathrm{g}$ HSA). The geometric mean serum antibody titer of OA-immunized animals was $1: 6,900$ and 1:2,200 for HSA-immunized animals. There was no detectable cross-agglutination on passive hemagglutination between the two antisera. The results in Fig. 3 show that immunization altered the pattern of OA absorption in a manner similar to that seen for HSA absorption. The TCA-precipitable fraction of ${ }^{125}$ I was significantly lower in the blood of OA-immunized lungs at $1 \mathrm{~h}$ and beyond $(P<0.001)$. At $4 \mathrm{~h}, \cong 9 \%$

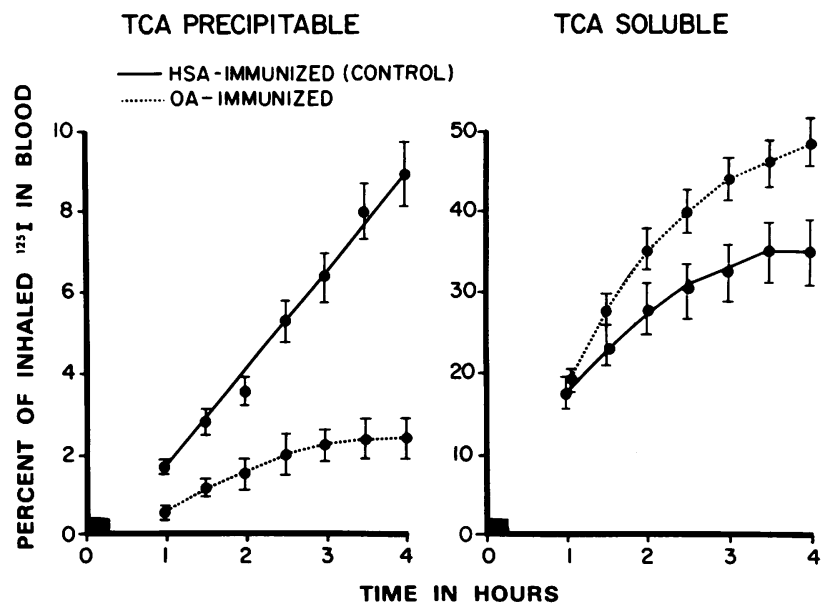

FIGURE 3 The appearance of TCA-precipitable and TCAsoluble ${ }^{125} \mathrm{I}$ in the blood of lungs insufflated with ${ }^{125} \mathrm{I}-\mathrm{OA}$. The dark bar represents 15 min of insufflation with ${ }^{125}$ I-OA. Each point represents the mean $\pm S E$ of the percent of inhaled ${ }^{125} \mathrm{I}$ in the blood of nine OA-immunized lungs and nine HSA-immunized controls. 
of the inhaled dose of ${ }^{125} \mathrm{I}$ was present in the blood of non-OA immune lungs in this fraction, whereas $<2.5 \%$ was found in the blood of OA-immune lungs, which is more than a threefold reduction. At $4 \mathrm{~h}$, TCAsoluble ${ }^{125} \mathrm{I}$ accounted for $35 \%$ of the dose in blood of non-OA-immune lungs and $48 \%$ in OA-immune lungs, which is almost $40 \%$ higher $(P<0.02)$.

There was no significant difference between any of the groups studied in physiologic parameters (blood pressure, edema) or amount of vapor inhaled. Also, $\mathrm{OA}$ introduced directly into the perfusing blood of control lungs (HSA-immune) did not decrease in concentration and was not metabolized.

The TCA-precipitable ${ }^{125}$ I found in the plasma was nondialyzable, precipitable with anti-OA serum but not with anti-HSA serum in a coprecipitation assay (Table I), and eluted in the void volume of a Sephadex G-10 column. Thin-layer chromatography of the TCAsoluble fraction revealed a heterogeneous pattern similar to that obtained when RISA was inhaled (Fig. 2).

Isolated lungs insufflated with rabbit serum albumin. Inasmuch as the absorption of OA from control lungs differed from that of HSA, especially the amount of TCA-soluble fraction which entered the blood, we also studied the absorption of homologous albumin from rabbit lungs. Rabbit serum albumin labeled with ${ }^{125}$ I was insufflated into isolated lungs from six nonimmune rabbits. The amounts of TCA-precipitable and TCA-soluble ${ }^{125} \mathrm{I}$ found in the blood at each time interval were not significantly different from those of normal lungs insufflated with RISA. At the end of $4 \mathrm{~h}$, the TCA-precipitable and TCA-soluble ${ }^{125} \mathrm{I}$ accounted for $6.7 \pm 0.7$ and $4.5 \pm 0.7 \% \mathrm{SE}$ of the inhaled dose, respectively.

\section{DISCUSSION}

The results of this study show that ${ }^{131}$ I from inhaled RISA entered the circulation of the isolated perfused rabbit lung as two fractions. The antigenically intact fraction was precipitable by TCA or anti-HSA serum and the other, TCA-soluble, may represent metabolites. The appearance of the antigen in the blood was greatly reduced in lung preparations from HSA-immunized animals. On the other hand, the appearance of the TCA-soluble fraction was apparently increased in the immunized preparations.

Most of the TCA-soluble fraction of blood ${ }^{131}$ I chromatographed differently from free iodide, suggesting that ${ }^{131} \mathrm{I}$ was bound to amino acids and(or) peptides. Since small labeled molecules were neither contaminants of the inhaled RISA nor produced by nebulization, it is probable that albumin was metabolized in the biologic system itself. TCA-soluble ${ }^{131}$ I could not be demonstrated when RISA was added directly to the perfusing blood, nor could it be detected in bron- choalveolar washings. Although this suggests that the lung parenchyma is the site of metabolism, one cannot rule out rapid equilibration of the small molecules with blood or interstitial fluid. In the normal (nonimmune) lung preparation, the TCA-soluble ${ }^{131} \mathrm{I}$ accounted for $48 \%$ of the total counts per minute in the blood after $4 \mathrm{~h}$. Schultz et al. (10), using isolated dog lungs, also found that after instillation of RISA into the bronchi about half of ${ }^{131}$ I which entered the blood was not protein bound. They suggested that RISA may break down in the lung. Other investigators studying the fate of intratracheally injected protein in vivo have reported that radioactivity in the blood was largely protein bound. Estimates range from 70 to $92 \%$ of blood radioactivity associated with the inhaled protein (11-13). This difference might be due to distribution of a small labeled molecule throughout the body, which is not a factor in the isolated lung. Thus, the present study presents the possibility that metabolism plays a significant role in the elimination of inhaled antigen from the isolated perfused lung and may be an important mechanism in vivo. Furthermore, immunization may enhance the metabolism of antigen in the lung.

Although we have not characterized the TCAprecipitable ${ }^{131}$ I recovered from blood, it is relevant that the molecule is nondialyzable and has retained its antigenicity. Schultz et al. (10) found that most of the protein bound ${ }^{131}$ I entering the blood of isolated dog lungs was associated with albumin, as identified by passage through DEAE-Sephadex. In contrast to the significant amount of antigenically intact ${ }^{131} \mathrm{I}$ which entered the blood of our normal rabbit lungs, only one-third to one-fourth as much appeared in the blood of immune lungs. This difference did not appear to be a nonspecific result of the immunization procedure per se, since the same procedure carried out with BGG did not cause a reduction in the appearance of RISA.

The changes in Ag absorption caused by immunization were also observed in experiments using a second antigen. Immunization resulted in a threefold decrease in the amount of antigenically intact $\mathrm{OA}$ and a $40 \%$ increase in the amount of metabolite in the circulation. The handling of OA by control lungs differed dramatically from that of HSA as there was a much more rapid initial clearance of TCA-soluble ${ }^{125} \mathrm{I}$. Approximately $80 \%$ more intact antigen and seven times as much metabolite (normalized to inhaled dose) was present in the blood of lungs insufflated with $\mathrm{OA}$ at the end of $4 \mathrm{~h}$. This represented a removal from the lung of $>40 \%$ of the inhaled dose of OA compared to 9\% of HSA removed in the same time period. A homologous serum protein, rabbit serum albumin, was metabolized to almost the same extent as RISA. The relatively rapid metabolism of OA by the lung is interesting, since others have reported that glycoproteins with certain 
terminal oligosaccharides, especially mannosyl residues (the structure shown for OA [14]), are cleared much faster by the liver than is pure protein $(15,16)$.

A number of mechanisms could account for an immunologically induced reduction in antigen absorption from the lung: (a) The lung of an immunized animal may have enhanced ability to metabolize specific antigen or protein in general. Our results suggest that metabolism of specific antigen was enhanced, since more of the TCA-soluble counts per minute were found in the blood of lungs immunized with that antigen. This may have been a contributing mechanism, especially in OA-immunized lungs. However, in HSAimmunized lungs the increased appearance of the TCA-soluble ${ }^{131}$ I was not large enough to account for the larger reduction in appearance of intact antigen. (b) A specific immunologic reaction could induce changes in pulmonary function (e.g. anaphylaxis) which would nonspecifically reduce the clearance of protein. Lungs from animals immunized with the homologous antigen did not undergo any obvious changes in pulmonary physiology as a result of antigen inhalation; there were no consistent differences in pulmonary arterial blood pressure, fluid gain, or quantity of antigen inhaled. However, more subtle alterations in pulmonary physiology cannot be excluded at this time. (c) A third possibility is that an immunologic mechanism in the lung creates a selective barrier to the normal transport of the antigen. Systemic immunization can result in the presence of intrapulmonary antibody, either by local synthesis, diffusion or selective transport from the serum $(17,18)$. Stokes et al. (4) blocked the absorption of RISA-dinitrophenol conjugate from rat lung by intratracheal injection of myeloma serum containing a high titer of anti-dinitrophenol IgA. This mechanism is appealing in light of a series of experiments by Walker et al. (19) on antigen absorption by the rat intestine. Small quantities of intact protein are normally absorbed into the bloodstream by the mammalian gut (20). Immunization (oral, intramuscular, or intraperitoneal) resulted in decreased absorption of horseradish peroxidase or bovine serum albumin by everted gut sacs. They demonstrated that IgGl antibodies on the luminal surface of the gut complex with antigen resulting in reduced binding of antigen to, and subsequent pinocytosis by, the intestinal epithelial cells. Furthermore, the antigen-antibody complexes remaining in the mucous coat were more rapidly degraded. Bensch and Dominguez (2) demonstrated that transalveolar movement of protein can occur in pinocytotic vesicles of the alveolar epithelial cells (type I pneumocytes) and the pulmonary capillary endothelium. The same transport mechanism exists in certain cells in the bronchial tract (21). Because the lung and gastrointestinal tract are both organs which sample large amounts of the environment, have many similarities in immune defense mechanisms against infectious agents (22-24) and may even have some mechanisms in common for protein absorption $(2,19)$, it would not be surprising to find similar defense mechanisms against the entry of potentially injurous antigens from the environment.

The fact that the isolated lungs showed little change in vasomotor tone, fluid uptake, or $\mathrm{pH}$ for an extended period, is a strong indication that the preparations were metabolically viable (25). However, one must interpret rates of transport by the isolated lung with caution as unknown factors may affect permeability, as well as the amount of antigen which reaches the alveoli. It should be noted, however, that the amount of RISA (mol wt $=69,000$ daltons) and OA (mol wt $=42,000$ daltons) in the blood of nonimmune preparations at the end of $4 \mathrm{~h}, \cong 5-9 \%$ of inhaled dose, respectively, was within the range of values reported for a similar interval by other investigators in vivo in the guinea pig (human gamma globulin, $\mathrm{mol} \mathrm{wt}=150,000$ ) (12), dog (RISA) (13), and rabbit (OA) (5). Dominguez et al. (3) reported much higher rates of RISA transport by the guinea pig lung. Regardless of absolute values, the air-blood barrier is a potential route of antigen absorption and, in the present study, the transport was greatly reduced by immunization. This may partially explain the observation of Thrall et al. (5) that less intratracheally instilled ${ }^{125} \mathrm{I}-\mathrm{OA}$ could be found in the blood of immunized rabbits than in the blood of normal animals.

In summary, the isolated perfused rabbit lung appears to provide a useful model for studying the mechanisms by which immunization alters the absorption of inhaled antigen across the air-blood barrier. In the nonimmunized lung, RISA was cleared at an appreciable rate as small molecules, possibly metabolites, while an equal amount entered the circulation antigenically intact. OA was cleared somewhat faster as antigen and was metabolized to a much greater extent. In both cases, the rate of appearance of intact antigen in the blood of normal lungs and its severalfold reduction in the blood of immune lungs were within ranges which could account for the observations of Thrall et al. (5) in vivo. This suggests that the air-blood barrier may be an important route of entry of inhaled antigen into the bloodstream, and that this route is at least partially blocked by immunization. In addition, immunization may accelerate the clearance of antigenic protein from the lung by metabolism.

\section{ACKNOWLEDGMENTS}

The authors wish to thank Mr. Tawfic S. Hakim for teaching Dr. Braley the technique of the isolated perfused rabbit lung. We are also indebted to Dr. David L. Roerig and Dr. Thad C. Hagen for their assistance in biochemical procedures and 
use of equipment, and to Mrs. Patricia L. Stevens for typing the manuscript.

This work was supported by grant HL 19733-01 from the National Heart, Lung and Blood Institute, the Medical Research Service of the Veterans Administration, and was aided by Specialized Center of Research (SCOR) grant HL 15389 from the National Institutes of Health.

\section{REFERENCES}

1. Morrow, P. E. 1972. Lymphatic drainage of the lung in dust clearance. Ann. N. Y. Acad. Sci. 200: 46-65.

2. Bensch, K. G., and E. A. M. Dominguez. 1971. Studies on the pulmonary air-tissue barrier. IV. Cytochemical tracing of macromolecules during absorption. Yale J. Biol. Med. 43: 236-241.

3. Dominguez, E. A. M., H. A. Liebow, and K. G. Bensch. 1967. Studies on the pulmonary air-tissue barrier. I. Absorption of albumin by the alveolar wall. Lab. Invest. 16: $905-1001$.

4. Stokes, C. R., J. F. Soothill, and M. W. Turner. 1975. Immune exclusion is a function of IgA. Nature (Lond.). 255: 745-748.

5. Thrall, R. S., L. B. Peterson, J. H. Linehan, P. Abramoff, and V.L. Moore. The effect of immunization on the uptake of intratracheally-administered antigen. Clin. Immunol. Immunopathol. In press.

6. Campbell, D. H., F. S. Garvey, N. E. Cremer, and D. H. Sussdorf. 1970. In Methods in Immunology. W. A. Benjamin, Inc., Menlo Park, Calif. 2nd edition. 246-250.

7. Bale, W. F., R. W. Helmkamp, T. P. Davis, M. J. Izzo, R. L. Goodland, M. A. Contreras, and I. L. Spar. 1966. High specific activity labeling of protein with ${ }^{131}$ I by the iodine monochloride method. Proc. Soc. Exp. Biol. Med. 122: 407-413.

8. Hakim, T. S., C. A. Dawson, V. L. Moore, and J. J. Barboriak. Anaphylaxis in isolated rabbit lung. Respiration. In press.

9. Mercer, T. 1973. Production and characterization of aerosols. Inhaled aerosol symposium. Arch. Intern. Med. 131: 39-50.

10. Schultz, A. L., J. T. Grismer, S. Wada, and F. Grande. 1964. Absorption of albumin from alveoli of perfused dog lung. Am. J. Physiol. 207: 1300-1304.

11. Bensch, K. G., E. Dominguez, and H. A. Liebow. 1967. Absorption of intact protein molecules across the pulmonary air-tissue barrier. Science (Wash. D. C.). 157: $1204-1206$.

12. Nash, D. R., and B. Holle. 1973. Local and systemic immune responses in guinea pigs given antigen parenterally or directly into the lower respiratory tract. Clin. Exp. Immunol. 13: 573-583.

13. Schultz, A. L., J. T. Grismer, and F. Grande. 1963. Absorption of radioactive albumin from the lungs of normal dogs. J. Lab. Clin. Med. 61: 494-500.

14. Narita, K. 1972. The N-terminal acetyl group of proteins and its possible implications for protein biosynthesis. In Proteins, Structure, and Function. M. Funatusa, K. Hirom, K. Imahori, T. Murachi, K. Narita, editors. John Wiley \& Sons, New York. 2: 227-239.

15. Winkelhake, J. L., and G. L. Nicolson. 1976. Aglycosylantibody: effects of exoglycosidase treatments on autochthonous antibody survival time in the circulation. J. Biol. Chem. 251: 1074-1080.

16. Baynes, J. W., and F. Wold. 1976. Effect of glycosylation on the in vivo circulating half-life of ribonuclease. $J$. Biol. Chem. 251: 6016-6024.

17. Kaltreider, H. B. 1976. Expression of immune mechanisms in the lung. Am. Rev. Respir. Dis. 113: 347-379.

18. Kazmierowski, J. A., W. A. Darbin, and H. Y. Reynolds. 1976. Kinetics of immunoglobulin transport into canine bronchial secretions. Proc. Soc. Exp. Biol. Med. 152: 493-498.

19. Walker, W. A., M. Wu, K. J. Isselbacher, and K. J. Bloch. 1975. Intestinal uptake of macromolecules. III. Studies on the mechanism by which immunization interferes with antigen uptake. J. Immunol. 115: 854-861.

20. Warshaw, A. L., W. A. Walker, and K. J. Isselbacher. 1974. Protein uptake by the intestine: evidence for absorption of intact molecules. Gastroenterology. 66: 987-992.

21. Richardson, J., T. Bouchard, and C. C. Ferguson. 1976. Uptake and transport of exogenous proteins by respiratory epithelium. Lab. Invest. 35: 307-314.

22. Bienenstock, J. 1974. The Physiology of the Local Immune Response and the G.I. Tract. In Progress in Immunology, II. L. Brent, and J. Holborow, editors. Elsevier North-Holland, Inc. New York. 4: 197-207.

23. Bienenstock, J., N. Johnston, and D. Y. E. Perey. 1973. Bronchial lymphoid tissue. II. Functional characteristics. Lab. Invest. 28: 693-698.

24. Rudzik, R., R. L. Clancy, D. Y. E. Perey, R. P. Day, and J. Bienenstock. 1976. Repopulation with IgA-containing cells of bronchial and intestinal lamina propria after transfer of homologous peyers patch and bronchial lymphocytes. J. Immunol. 114: 1599-1604.

25. Niemeier, G., and R. W. Bingham. 1972. An isolated perfused lung preparation for metabolic studies. Life Sci. 11: 807-820. 